\title{
WEAK UPPER TOPOLOGIES AND DUALITY FOR CONES
}

\author{
KLAUS KEIMEL
}

Fachbereich Mathematik, Technische Universität Darmstadt, Schloßgartenstraße 7, D-64289 Darmstadt, Germany

e-mail address: keimel@mathematik.tu-darmstadt.de

\begin{abstract}
In functional analysis it is well known that every linear functional defined on the dual of a locally convex vector space which is continuous for the weak* topology is the evaluation at a uniquely determined point of the given vector space. M. Schröder and A. Simpson have obtained a similar result for lower semicontinuous linear functionals on the cone of all Scott-continuous valuations on a topological space endowed with the weak* upper topology, an asymmetric version of the weak* topology. This result has given rise to several proofs, originally by Schröder and Simpson themselves and, more recently, by the author of these Notes and by J. Goubault-Larrecq. The proofs developed from very technical arguments to more and more conceptual ones. The present Note continues on this line, presenting a conceptual approach inspired by classical functional analysis which may prove useful in other situations.
\end{abstract}

\section{INTRODUCTION}

Recently, a theorem announced by Schröder and Simpson in [15] and presented with a proof in [16] has attracted some attention. Before stating this result we need some preparations.

Let $X$ be an arbitrary topological space. The emphasis is on non-Hausdorff spaces like directed complete partially ordered sets (= dcpos) with the Scott topology (see [4]) and quotients of countably based spaces (qcb-spaces, see [2]). But, of course, compact and locally compact Hausdorff spaces are classical examples. We consider functions defined on $X$ with values in $\overline{\mathbb{R}}_{+}$, the set $\mathbb{R}_{+}$of nonnegative real numbers extended by $+\infty$ as top element. Such functions $f$ are lower semicontinuous if, for every $r \in \mathbb{R}_{+}$, the set $\{x \in X \mid f(x)>r\}$ is open in $X$. We denote by $\mathcal{L} X$ the set of all lower semicontinuous functions $f: X \rightarrow \overline{\mathbb{R}}_{+}$with the pointwise defined order. The pointwise supremum of any family of lower semicontinuous functions is lower semicontinuous so that $\mathcal{L} X$ is a complete lattice. The pointwise defined sum $f+g$ of two lower semicontinuous functions $f, g$ as well as the scalar multiple $r f$ for $r \in \mathbb{R}_{+}$are also lower semicontinuous.

2012 ACM CCS: [Mathematics of computing]: Mathematical analysis - Functional analysis; [Theory of computation]: Semantics and reasoning-Program semantics-Denotational semantics.

Key words and phrases: Semitopological cones, directed complete partially ordered cones, weak upper topologies, dual cones, Schröder-Simpson Theorem.

Supported by DFG (Deutsche Forschungsgemeinschaft). 
The maps $\mu: \mathcal{L} X \rightarrow \overline{\mathbb{R}}_{+}$which are linear and Scott-continuous, that is, those satisfying

$$
\mu(f+g)=\mu(f)+\mu(g), \quad \mu(r f)=r \mu(f), \quad \mu\left(\bigvee_{i}^{\uparrow} f_{i}\right)=\bigvee_{i}^{\uparrow} \mu\left(f_{i}\right)
$$

for all $f, g \in \mathcal{L} X, r \in \mathbb{R}_{+}$and for every directed family $\left(f_{i}\right)_{i}$ in $\mathcal{L} X$, form the valuation powerdomain $\mathcal{V} X$. On $\mathcal{V} X$ one also has a pointwise defined order, addition and multiplication by nonnegative real numbers. We endow $\mathcal{V} X$ with the weak*upper topology, that is, the coarsest topology such that the evaluation maps $\mu \mapsto \mu(f): \mathcal{V} X \rightarrow \overline{\mathbb{R}}_{+}$remain lower semicontinuous for all $f \in \mathcal{L} X$. The Schröder-Simpson theorem says:

Theorem 1.1. For every linear functional $\varphi: \mathcal{V} X \rightarrow \overline{\mathbb{R}}_{+}$which is lower semicontinuous with respect to the weak $k^{*}$ upper topology, there is an $f \in \mathcal{L} X$ such that $\varphi(\mu)=\mu(f)$ for all $\mu \in \mathcal{V} X$.

This theorem sounds exactly like a simple general fact from classical functional analysis (see, e.g., [14, Chapter IV, 1.2]): Let $V$ be a topological vector space over the field of real numbers and $V^{*}$ the dual vector space of all continuous linear functionals $\mu: V \rightarrow \mathbb{R}$, then every linear functional $\varphi$ defined on the topological dual $V^{*}$ which is continuous with respect to the weak* topology is the evaluation at some $f \in V$, that is, $\varphi(\mu)=\mu(f)$ for every $\mu \in V^{*}$. Here the weak* topology is the coarsest topology on $V^{*}$ such that all the evaluation maps $\mu \rightarrow \mu(f): V^{*} \rightarrow \mathbb{R}, f \in V$, remain continuous. (Here $\mathbb{R}$ is meant to carry the usual Hausdorff topology). The idea behind the quite simple proof of this classical result is to reduce the question to the finite dimensional case, where it is trivial.

The Schröder-Simpson Theorem is a kind of asymmetric version of this classical fact in a special situation. Its original proof was technical and involved. A new proof in a conceptual framework was published in [9]. A short proof by J. Goubault-Larrecq [5] has appeared just recently. Let us remark that the Schröder-Simpson theorem has a predecessor in Domain Theory: If $X$ is a continuous dcpo (with the Scott topology), then $\mathcal{V} X$ is a continuous dcpo, too, and every Scott-continuous linear functional on $\mathcal{V} X$ is the evaluation at some $f \in \mathcal{L} X$ (see C. Jones [7] and Kirch [11]). For a continuous dcpo $X$, the Scott topology on $\mathcal{V} X$ agrees with the weak*upper topology as Kirch [11] has shown, a fact that is now longer true for more general spaces. Thus, the Schröder-Simpson Theorem is a far reaching generalization.

The proofs of the Schröder-Simpson theorem due to Keimel and Goubault-Larrecq are based on two lemmas that hold for every lower semicontinuous linear functional $\varphi$ from the valuation powerdomain $\mathcal{V} X$ with the weak*upper topology into $\overline{\mathbb{R}}_{+}$, where $X$ is any topological space:

Lemma 1.2. (Lemma 2 in [5], Theorem 5.3 in [9]) There is a family $\left(f_{i}\right)_{i}$ of functions in $\mathcal{L} X$ such that $\varphi(\mu)=\sup _{i} \mu\left(f_{i}\right)$ for every $\mu \in \mathcal{V} X$.

Lemma 1.3. (Lemma 1 in [5], Lemma 5.5 in [9]) The set of all $f \in \mathcal{L} X$ such that $\varphi(\mu) \geq$ $\mu(f)$ for all $\mu \in \mathcal{V} X$ is directed.

The Schröder-Simpson Theorem 1.1 can now be deduced as follows: Given a lower semicontinuous linear $\varphi: \mathcal{V} X \rightarrow \overline{\mathbb{R}}_{+}$, Lemma 1.2 gives us a family of $\left(f_{i}\right)_{i}$ of functions in $\mathcal{L} X$ such that $\varphi(\mu)=\sup _{i} \mu\left(f_{i}\right)$ for all $\mu \in \mathcal{V} X$. By Lemma 1.3 the family $\left(f_{i}\right)_{i}$ can be chosen to be directed and we let $f=\sup _{i} f_{i}$. Since the valuations $\mu \in \mathcal{V} X$ are Scott-continuous on $\mathcal{L} X$, they preserve directed suprema and we obtain that $\varphi(\mu)=\sup _{i} \mu\left(f_{i}\right)=\mu\left(\sup _{i} f_{i}\right)=\mu(f)$ for all $\mu \in \mathcal{V} X$. In fact, it suffices to find a subcone $C$ of $\mathcal{L} X$ and to prove the two lemmas above for subfamilies of $C$, for example for the subcone of step functions. 
It is desirable to prove an analogue of the Schröder-Simpson Theorem for situations different from the valuation powerdomain. For the moment I do not know how to generalize Lemma 1.3. For Lemma 1.2, a general conceptual argument had been developed in [9, Section 5.1] by reducing the problem to a situation close to the classical vector space situation; but this argument needed some quite heavy background from the theory of quasi-uniform locally convex cones. Goubault-Larrecq found a simple direct argument for the proof of Lemma 1.2 for the special situation of the valuation powerdomain. In this note we present a direct approach to Lemma 1.2 based on Goubault-Larrecq's idea but in an appropriate generality. It uses the classical idea to reduce the problem to the finite dimensional case.

Let us recall the motivation behind all of this. The Schröder-Simpson theorem concerns the probabilistic powerdomain over arbitrary topological spaces used in semantics for modelling probabilistic phenomena in programming. Goubault-Larrecq [6] recently has applied the result in his work on modelling probabilistic and ordinary nondeterminism simultaneously.

\section{Cones And separation}

The Schröder-Simpson Theorem cannot be proved with methods from classical functional analysis, since the real-valued lower semicontinuous functions on a topological space $X$ do not form a vector space. For a lower semicontinuous function $f: X \rightarrow \mathbb{R}$, its negative $-f$ is

not necessarily lower semicontinuous. Similarly, we are in an asymmetric situation for the valuations, those being Scott-continuous linear functionals. We therefore work with cones, a weakening of the notion of a vector space by restricting scalar multiplication to nonnegative reals and not requiring the existence of additive inverses. This offers the advantage of including infinity. In this section we provide some basic concepts. The connection to our goal will appear only later.

We denote by $\mathbb{R}_{+}$the set of nonnegative real numbers with the usual linear order, addition and multiplication. The letters $r, s, t, \ldots$ will always denote nonnegative reals. Further

$$
\overline{\mathbb{R}}_{+}=\mathbb{R}_{+} \cup\{+\infty\}
$$

denotes the nonnegative reals extended by $+\infty$ as a top element. We also extend addition and multiplication from $\mathbb{R}_{+}$to $\overline{\mathbb{R}}_{+}$by defining $r+\infty=+\infty+r=+\infty$ for all $r \in \overline{\mathbb{R}}_{+}$and

$$
r \cdot(+\infty)=+\infty \cdot r= \begin{cases}+\infty & \text { for } r>0 \\ 0 & \text { for } r=0\end{cases}
$$

Definition 2.1. A cone is a commutative monoid $C$ carrying a scalar multiplication by nonnegative real numbers satisfying the same axioms as for vector spaces; that is, $C$ is endowed with an addition $(x, y) \mapsto x+y: C \times C \rightarrow C$ and a neutral element 0 satisfying:

$$
\begin{aligned}
x+(y+z) & =(x+y)+z \\
x+y & =y+x \\
x+0 & =x
\end{aligned}
$$


and with a scalar multiplication $(r, x) \mapsto r \cdot x: \mathbb{R}_{+} \times C \rightarrow C$ satisfying:

$$
\begin{aligned}
r \cdot(x+y) & =r \cdot x+r \cdot y \\
(r+s) \cdot x & =r \cdot x+s \cdot x \\
(r s) \cdot x & =r \cdot(s \cdot x) \\
1 \cdot x & =x \\
0 \cdot x & =0
\end{aligned}
$$

for all $x, y, z \in C$ and all $r, s \in \mathbb{R}_{+}$. An ordered cone is a cone $C$ endowed with a partial order $\leq$, such that addition and multiplication by fixed scalars $r \in \mathbb{R}_{+}$are order preserving, that is, for all $x, y, z \in C$ and all $r \in \mathbb{R}_{+}$:

$$
x \leq y \Longrightarrow x+z \leq y+z \text { and } r \cdot x \leq r \cdot y
$$

Unlike for vector spaces, addition in cones need not satisfy the cancellation property, and cones need not be embeddable in vector spaces. For example $\overline{\mathbb{R}}_{+}=\mathbb{R}_{+} \cup\{+\infty\}$ is an ordered cone that is not embeddable in a vector space. The same holds for finite powers $\overline{\mathbb{R}}_{+}^{n}$ and for infinite powers $\overline{\mathbb{R}}_{+}^{I}$ with the pointwise defined order $x \leq y$ iff $x_{i} \leq y_{i}$ for all indices $i$. Maps $\varphi$ from a cone $C$ into $\overline{\mathbb{R}}_{+}$are simply referred to as functionals. Thus, functionals are allowed to have the value $+\infty$. On any set of functionals we consider the pointwise order $\varphi \leq \psi$ if $\varphi(x) \leq \psi(x)$ for all $x \in C$.

Definition 2.2. Let $C$ be a cone. A functiona 1$] \varphi: C \rightarrow \overline{\mathbb{R}}_{+}$is called

$$
\begin{aligned}
& \text { homogeneous, if: } \quad \varphi(r \cdot a)=r \cdot \varphi(a) \quad \text { for all } a \in C \text { and all } r \in \mathbb{R}_{+} \text {, } \\
& \text { additive, if: } \quad \varphi(a+b)=\varphi(a)+\varphi(b) \text { for all } a, b \in C \text {, } \\
& \text { superadditive if: } \varphi(a+b) \geq \varphi(a)+\varphi(b) \text { for all } a, b \in C \text {, } \\
& \text { subadditive if: } \quad \varphi(a+b) \leq \varphi(a)+\varphi(b) \text { for all } a, b \in C \text {. }
\end{aligned}
$$

We say that $\varphi$ is sublinear (superlinear, linear, respectively), if it is homogeneous and subadditive (superadditive, additive, respectively).

As in real vector spaces, we have a notion of convexity:

Definition 2.3. A subset $K$ of a cone $C$ is convex if, for all $a, b \in K$, the convex combinations $r a+(1-r) b$ belong to $K$ for all real number $r$ with $0 \leq r \leq 1$.

Convex sets in cones may look different than those in vector spaces because of the presence of infinite elements. Indeed, in $\overline{\mathbb{R}}_{+}$not only intervals $\mathbb{I}$ are convex but also the sets $\mathbb{I} \cup\{+\infty\}$.

To every functional $\varphi: C \rightarrow \overline{\mathbb{R}}_{+}$we assign the sets

$$
U_{\varphi}=\{x \in C \mid \varphi(x)>1\}, \quad A_{\varphi}=\{x \in C \mid \varphi(x) \leq 1\}
$$

which are complements one of the other. These sets reflect properties of the functionals:

Remark 2.4. For homogeneous functionals $\varphi$ and $\psi$ on a cone $C$ we have:

(a) $\varphi \leq \psi$ if and only if $U_{\varphi} \subseteq U_{\psi}$ if and only if $A_{\varphi} \supseteq A_{\psi}$.

Indeed, $\psi \leq \varphi$ iff, for all $0<r<+\infty, \psi(x) \leq r \Longrightarrow \varphi(x) \leq r$. For $0<r<+\infty$, $\psi(x) \leq r \Longrightarrow \varphi(x) \leq r$ iff $\psi\left(\frac{x}{r}\right) \leq 1 \Longrightarrow \varphi\left(\frac{x}{r}\right) \leq 1$ iff $\frac{x}{r} \in A_{\psi} \Longrightarrow \frac{x}{r} \in A_{\varphi}$ iff $x \in r A_{\psi} \Longrightarrow x \in r A_{\varphi}$ iff $r A_{\psi} \subseteq r A_{\varphi}$ iff $A_{\psi} \subseteq A_{\varphi}$.

\footnotetext{
${ }^{1}$ We simply call homogeneous what is usually called positively homogeneous. Previously authors always supposed $\varphi(0)=0$ for homogeneous functionals; note that we omit this requirement.
} 
(b) If $\varphi$ is sublinear then $A_{\varphi}$ is convex.

Indeed, if $\varphi$ is sublinear and $a, b$ are elements in $A_{\varphi}$, then $\varphi(r a+(1-r) b) \leq \varphi(r a)+\varphi((1-$ $r) b)=r \varphi(a)+(1-r) \varphi(b) \leq 1$, whence $r a+(1-r) b \in A_{\varphi}$.

(c) If $\psi$ is superlinear then $U_{\psi}$ is convex.

The following technical result is the key to our main result:

Lemma 2.5. Let $V=\left\{\left(y_{1}, \ldots, y_{n}\right) \in \overline{\mathbb{R}}_{+}^{n} \mid y_{i}>1\right.$ for all $\left.i=1, \ldots, n\right\}$ and $K$ be a convex subset in $\overline{\mathbb{R}}_{+}^{n}$ disjoint from $V$. Then there are nonnegative real numbers $a_{1}, \ldots, a_{n}$ such that $\sum_{i=1}^{n} a_{i}=1$ and

$$
\sum_{i} a_{i} x_{i} \leq 1<\sum_{i} a_{i} y_{i} \quad \text { for all }\left(x_{1}, \ldots, x_{n}\right) \in K,\left(y_{1}, \ldots, y_{n}\right) \in V
$$

Proof. Let $V$ and $K$ be as in the statement of the lemma. The convex hull of $K \cup[0,1]^{n}$ does not meet $V$. Indeed, if $x \in K$, then $x_{i} \leq 1$ for some coordinate $x_{i}$ of $x$; as $y_{i} \leq 1$ for every coordinate of $y \in[0,1]^{n}$, the $i$-th coordinate of every convex combination of $x$ and $y$ is $\leq 1$. As $V$ is an upper set, the lower set $L$ of all elements $z \in \overline{\mathbb{R}}_{+}^{n}$ that are below some element in the convex hull of $K \cup[0,1]^{n}$ does not meet $V$, and $L$ is convex, too.

We now consider the convex subset $L^{\prime}=L \cap \mathbb{R}^{n}$ and the convex open subset $V^{\prime}=$ $V \cap \mathbb{R}^{n}$ of $\mathbb{R}^{n}$ which are disjoint. By a standard separation theorem (see the remark below), there is a linear functional $f: \mathbb{R}^{n} \rightarrow \mathbb{R}$ such that $f(x) \leq 1<f(y)$ for all $x \in L^{\prime}$ and all $y \in V^{\prime}$. As for every linear functional on $\mathbb{R}^{n}$, there are real numbers $a_{1}, \ldots, a_{n}$ such that $f\left(x_{1}, \ldots, x_{n}\right)=\sum_{i} a_{i} x_{i}$ for every $x=\left(x_{1}, \ldots, x_{n}\right) \in \mathbb{R}^{n}$. Let us show that $a_{i} \geq 0$ for every $i$ and $\sum_{i} a_{i}=1$.

Since $(1,1, \ldots, 1) \in L^{\prime}$, we have $\sum_{i} a_{i}=f(1,1, \ldots, 1) \leq 1$. Since $(1+\varepsilon, \ldots, 1+\varepsilon) \in V^{\prime}$ for every $\varepsilon>0$, we have $\sum_{i} a_{i}+n \varepsilon=f(1+\varepsilon, \ldots, 1+\varepsilon)>1$ for all $\varepsilon>0$, whence $\sum_{i} a_{i} \geq 1$. We finally show that $a_{1} \geq 0$. Since the vector $(2+\varepsilon, 1+\varepsilon, \ldots, 1+\varepsilon)$ belongs to $V^{\prime}$ for every $\varepsilon>0$, we have $1<f(2+\varepsilon, 1+\varepsilon, \ldots, 1+\varepsilon)=a_{1}+\sum_{i}^{n} a_{i}+n \varepsilon=a_{1}+1+n \varepsilon$, that is, $0<a_{1}+n \varepsilon$ for every $\varepsilon>0$, which implies $a_{1} \geq 0$. Similarly, $a_{i} \geq 0$ for all $i$.

The restriction of $f$ to the positive cone $\mathbb{R}_{+}^{n}$ has a unique Scott-continuous extension to $\overline{\mathbb{R}}_{+}^{n}$ also given by $\sum_{i} a_{i} x_{i}$ for all $x=\left(x_{1}, \ldots, x_{n}\right) \in \overline{\mathbb{R}}_{+}^{n}$ which has the desired separation property for $K$ and $V$.

Remark 2.6. In the proof of the preceding Lemma 2.5 we have used a standard Separation Theorem which can be proved for arbitrary topological vector spaces $E$ over the reals (see, e.g., [13, Theorem 3.4(a)]): For every open convex subset $V^{\prime}$ and every convex subset $L^{\prime}$ disjoint from $V^{\prime}$ there are a continuous linear functional $f: E \rightarrow \mathbb{R}$ and a real number $r$ such that $f(x) \leq r<f(y)$ for every $x \in L^{\prime}$ and every $y \in V^{\prime}$. Since $r \neq 0$ in the situation in the proof above, one may choose $r=1$ by replacing $f$ by $\frac{1}{r} f$.

The proof of this standard Separation Theorem uses the axiom of choice. We only need this Separation Theorem for finite dimensional real vector spaces. For this special case, there exist constructive proofs. One of the referees for this paper has worked out such a constructive proof. One may also use a separation theorem for convex subsets in finite dimensional vector spaces (see Aliprantis and Border [1, Theorem 7.35, p. 279]) often attributed to Minkowski. In 3], K. C. Border discusses the same result under the name of 'Finite Dimensional Separating Hyperplane Theorem 11', and Border's proof looks constructive, as far as I can see. Thus, our Lemma2.5 has a constructive proof, not using the axiom of choice or any weak form thereof, and the same holds for the following proposition. 
I am indebted to one of the referees for a detailed discussion on the issue of a constructive proof. Moreover, the proof of Lemma 2.5 has been simplified by a suggestion of this referee, namely to replace $K$ by the convex hull of $K$ with the cube $[0,1]^{n}$.

The following key result uses the reduction to the finite dimensional case as indicated in the Introduction:

Proposition 2.7. Consider a cone $D$ together with a sublinear functional $\varphi: D \rightarrow \overline{\mathbb{R}}_{+}$and finitely many linear functionals $\psi_{i}: D \rightarrow \overline{\mathbb{R}}_{+}, i=1, \ldots, n$, such that $\psi_{1} \wedge \cdots \wedge \psi_{n} \leq \varphi$. Then there are nonnegative real numbers $a_{1}, \ldots, a_{n}$ such that $\sum_{i} a_{i}=1$ and $\psi_{1} \wedge \cdots \wedge \psi_{n} \leq$ $\sum_{i=1}^{n} a_{i} \psi_{i} \leq \varphi$.

Proof. Let $A=A_{\varphi}$ be the set of all $x \in D$ such that $\varphi(x) \leq 1$. Since $\varphi$ is supposed to be sublinear, $A$ is convex. Further let $U$ be the set of all $x \in D$ such that $\left(\psi_{1} \wedge \cdots \wedge \psi_{n}\right)(x)=$ $\min _{i} \psi_{i}(x)>1$. Consider the map $\Psi: D \rightarrow \overline{\mathbb{R}}_{+}^{n}$ defined by $\Psi(x)=\left(\psi_{1}(x), \ldots, \psi_{n}(x)\right)$. This map $\Psi$ is linear, since all the $\psi_{i}$ are linear . For $x \in U$ we have $\psi_{i}(x)>1$ for every $i$, that is $\Psi(x) \in V=\left\{\left(x_{1}, \ldots, x_{n}\right) \in \overline{\mathbb{R}}_{+} \mid x_{i}>1, i=1, \ldots, n\right\}$. Since $\Psi$ is linear, the image $K=\Psi(A)$ of $A$ is convex. For $a \in A$, we have $\left(\psi_{1} \wedge \cdots \wedge \psi_{n}\right)(a) \leq \varphi(a) \leq 1$, whence $\left(\psi_{1}(a), \ldots, \psi_{n}(a)\right) \notin V$. Thus, $K=\Psi(A)$ is a convex subset of $\overline{\mathbb{R}}_{+}^{n}$ disjoint from $V$. By the Separation Lemma 2.5 there are nonnegative real numbers $a_{1}, \ldots, a_{n}$ such that $\sum_{i} a_{i}=1$ and $\sum_{i} a_{i} c_{i} \leq 1<\sum_{i} a_{i} d_{i}$ for all $c \in K$ and all $d \in V$. We denote by $G: \overline{\mathbb{R}}_{+}^{n} \rightarrow \overline{\mathbb{R}}_{+}$ the linear map $G(x)=\sum_{i} a_{i} x_{i}$. Then, on one hand, $G \circ \Psi$ is a linear functional on $D$. If $\left(\psi_{1} \wedge \cdots \wedge \psi_{n}\right)(x)>1$, then $x \in U$, hence $\Psi(x) \in V$ which implies $G(\Psi(x))>1$, whence $\psi_{1} \wedge \cdots \wedge \psi_{n} \leq G \circ \Psi$ by Remark 2.4(a). On the other hand, if $\varphi(a) \leq 1$, then $a \in A$, whence $G(\Psi(a)) \leq 1$; thus $G \circ \Psi \leq \varphi$ again by Remark 2.4(a). Noticing that, $G(\Psi(x))=\sum_{i=1}^{n} a_{i} \psi_{i}(x)$, that is, $G \circ \Psi=\sum_{i=1}^{n} a_{i} \psi_{i}$, we have the desired result.

In the above lemma, the maps $\psi_{i}$ are supposed to be linear; so the linear combination $\sum_{i} a_{i} \psi_{i}$ is linear, too. In particular, we have interpolated a linear functional between $\varphi$ and $\psi_{1} \wedge \cdots \wedge \psi_{n}$. Under the hypotheses of the preceding lemma, there are general separation theorems that yield directly the existence of a linear functional $\psi$ between the sublinear functional $\varphi$ and the superlinear functional $\psi_{1} \wedge \cdots \wedge \psi_{n}$. Our Lemma above gives us more information as it tells us that $\psi$ may be chosen to be a convex combination of the $\psi_{i}$ and this property will be crucial in the following.

One may prove the preceding lemma also under the weaker hypothesis that the maps $\psi_{i}$ are only superlinear and not linear. But we have no use for this generalization.

\section{Semitopological COnes And lower Semicontinuous functionals}

Every partially ordered set $P$ can be endowed with a simple topology, the upper topology $\nu$ : a subbasis for the closed sets is given by the principal ideals $\downarrow x=\{y \in P \mid y \leq x\}, x \in P$.

If not specified otherwise, we will use the upper topology on $\mathbb{R}_{+}$and on $\overline{\mathbb{R}}_{+}$. The proper nonempty open subsets are simply the open upper intervals $] r,+\infty\left[=\{x \mid x>r\}\right.$ on $\mathbb{R}_{+}$ and the open upper intervals $] r,+\infty]$ on $\overline{\mathbb{R}}_{+}$, where $r \in \mathbb{R}_{+}$.

This asymmetric upper topology on $\overline{\mathbb{R}}_{+}$is appropriate to talk about lower semicontinuity. In agreement with classical analysis, for an arbitrary topological space $X$, we call 
lower semicontinuou 2 those functions $f: X \rightarrow \overline{\mathbb{R}}_{+}$which are continuous with respect to the upper topology on $\overline{\mathbb{R}}_{+}$. Extended addition and multiplication are lower semicontinuous as maps $\overline{\mathbb{R}}_{+} \times \overline{\mathbb{R}}_{+} \rightarrow \overline{\mathbb{R}}_{+}$also at infinity.

For topological spaces $X, Y, Z$, a function $f: X \times Y \rightarrow Z$ will be said to be separately continuous if, for each fixed $x \in X$, the function $y \mapsto f(x, y): Y \rightarrow Z$ and, for each fixed $y \in Y$, the function $x \mapsto f(x, y): X \rightarrow Z$ is continuous. We say that $f$ is jointly continuous if it is continuous for the product topology on $X \times Y$.

Definition 3.1. A semitopological cone is cone equipped with a topology satisfying the $\mathrm{T}_{0}$ separation axiom such that addition $(x, y) \mapsto x+y: C \times C \rightarrow C$ and scalar multiplication $(r, x) \mapsto r x: \mathbb{R}_{+} \times C \rightarrow C$ are separately continuous. If these maps are jointly continuous, we have a topological cone.

Example 3.2. For a nonnegative integer $n$, clearly $\overline{\mathbb{R}}_{+}^{n}$ is a cone ordered by the coordinatewise order. The upper topology on $\overline{\mathbb{R}}_{+}^{n}$ is the product of the upper topology on $\overline{\mathbb{R}}_{+}$. Addition and extended scalar multiplication are continuous for the upper topology on $\overline{\mathbb{R}}_{+}^{n}$ so that $\overline{\mathbb{R}}_{+}^{n}$ is a topological cone. The same holds for infinite powers $\overline{\mathbb{R}}_{+}^{I}$.

Any topological $\mathrm{T}_{0}$ space $X$ comes with an intrinsic partial order, the specialization preorder defined by $x \leq y$ if the element $x$ is contained in the closure of the singleton $\{y\}$ or, equivalently, if every open set containing $x$ also contains $y$. In this paper, an order on a topological space will always be the specialization order. Open sets are upper $(=$ saturated) sets and closed sets are lower sets with respect to the specialization preorder. The saturation $\uparrow A$ of a subset $A$ can also be characterized to be the intersection of the open sets containing $A$. In Hausdorff spaces, the specialization order is trivial. On $\overline{\mathbb{R}}_{+}$, the specialization order for the upper topology agrees with the usual linear order. Continuous maps between topological $\mathrm{T}_{0}$ spaces preserve the respective specialization preorders. For more details, see e.g. 4, Section 0-5.

As we use the upper topology on $\mathbb{R}_{+}$, a semitopological cone $C$ cannot satisfy the Hausdorff separation property: Since continuous maps preserve the respective specialization preorders, the continuity of scalar multiplication implies firstly that, for every $a \in C$, the map $r \mapsto r a: \mathbb{R}_{+} \rightarrow C$ is order preserving, that is, the rays in the cone are linearly ordered, and secondly that the cone is pointed; indeed, $0=0 \cdot a \leq 1 \cdot a=a$ for all elements $a$ of the cone. In particular, the specialization order on $C$ is nontrivial and the topology is far from being Hausdorff except for the trivial case $C=\{0\}$. As continuous maps preserve the specialization order, we conclude that every semitopological cone is an ordered cone with bottom element 0 .

Let $D$ be a semitopological cone. There is a close relation between classes of open subsets of $D$ and classes of lower semicontinuous functionals on $D$.

An arbitrary functional $\varphi: D \rightarrow \overline{\mathbb{R}}_{+}$is lower semicontinuous if and only if, for every $r \in \mathbb{R}_{+}$, the set $\{x \in D \mid \varphi(x)>r\}$ is open. For a homogeneous functional this simplifies: A homogeneous functional is lower semicontinuous 3 if and only if the set

$$
U_{\varphi}=\{y \in D \mid \varphi(y)>1\}
$$

\footnotetext{
${ }^{2}$ It is somewhat unfortunate that those functions are called lower semicontinuous which are continuous with respect to the upper topology. But we do not want to deviate from the terminology in classical analysis and the one adopted in 4 .

${ }^{3}$ Homogeneous lower semicontinuous functionals on cones of lower semicontinuous maps are called previsions by Goubault-Larrecq.
} 
is open. Since $\varphi(0)=0$, the open set $U_{\varphi}$ does not contain 0. Conversely, for any open subset $U \subseteq D$ containing 0, the upper Minkowski functional

$$
\varphi_{U}(y)=\sup \{r>0 \mid y \in r \cdot U\}
$$

is lower semicontinuous and homogeneous; it is understood that $\varphi_{U}(x)=0$, if $x \notin r U$ for all $r>0$.

For any nonempty finite family $\varphi_{1}, \ldots, \varphi_{n}$ of homogeneous lower semicontinuous functions the pointwise infimum

$$
\left(\varphi_{1} \wedge \cdots \wedge \varphi_{n}\right)(x)=\min \left(\varphi_{1}(x), \ldots, \varphi_{n}(x)\right)
$$

is again homogeneous and lower semicontinuous. The same holds for the pointwise supremum $\left(\bigvee_{i} \varphi_{i}\right)(x)=\sup _{i} \varphi_{i}(x)$ of an arbitrary family $\left(\varphi_{i}\right)_{i}$ of homogeneous lower semicontinuous functionals.

We have the following properties sharpening Remark 2.4 (see [8, Propositions 7.4 and 7.5] and [12]):

\section{Lemma 3.3.}

(a) The assignment $\varphi \mapsto U_{\varphi}$ establishes an order isomorphism between the collection $\mathcal{H} X$ of all lower semicontinuous homogeneous functionals $\varphi: D \rightarrow \overline{\mathbb{R}}_{+}$ordered pointwise and the collection $\mathcal{O}^{*} X$ of all proper open subsets $U$ of $D$ ordered by inclusion; the inverse map is given by assigning to every $U \in \mathcal{O}^{*} X$ its Minkowski functional $\varphi_{U}(x)$. For homogeneous lower semicontinuous functionals we have:

$$
\begin{aligned}
\varphi \leq \psi & \Longleftrightarrow U_{\varphi} \subseteq U_{\psi}, \\
U_{\varphi_{1}} \cap \cdots \cap U_{\varphi_{n}} & =U_{\varphi_{1} \wedge \cdots \wedge \varphi_{n}}, \\
\bigcup_{i} U_{\varphi_{i}} & =U_{\left(\bigvee_{i} \varphi_{i}\right)} .
\end{aligned}
$$

(b) A homogeneous functional $\varphi$ is superlinear if and only if the corresponding open set $U_{\varphi}$ is convex.

(c) A homogeneous functional $\varphi$ is sublinear if and only if the complement $A_{\varphi}=D \backslash U_{\varphi}$ of the corresponding open set is convex.

\section{WEAK UPPER TOPOLOGIES AND THE MAIN RESULT}

We fix a dual pair of cones which, by definition, consists of two cones $C$ and $D$ together with a bilinear map $(x, y) \mapsto\langle x, y\rangle: C \times D \rightarrow \overline{\mathbb{R}}_{+}$. Bilinearity means that for each $x \in C$, the map

$$
\widehat{x}=(y \mapsto\langle x, y\rangle): D \rightarrow \overline{\mathbb{R}}_{+}
$$

is linear and similarly for fixed $y \in D$. We will always suppose this bilinear map to be non-singular, that is, for any two different elements $y \neq y^{\prime} \in D$ there is an $x \in C$ such that $\langle x, y\rangle \neq\left\langle x, y^{\prime}\right\rangle$.

As before, we endow $\overline{\mathbb{R}}_{+}$with the upper topology. We consider the coarsest topology $w(D, C)$ on $D$ rendering lower semicontinuous the maps $\widehat{x}=(y \mapsto\langle x, y\rangle): D \rightarrow \overline{\mathbb{R}}_{+}$for all $x \in C$. We call it the weak upper topology on $D$. A subbasis of this topology is given by the sets

$$
U_{x}=\{y \in D \mid \widehat{x}(y)=\langle x, y\rangle>1\}, \quad x \in C .
$$


Addition and scalar multiplication are jointly continuous for the weak upper topology $w(D, C)$ so that $D$ with the weak upper topology is a topological cone. Alternatively, $D$ may be mapped into the product space $\overline{\mathbb{R}}_{+}^{C}$ via the map $x \mapsto \widehat{x}$. The weak upper topology $w(D, C)$ is the topology induced by the upper topology on the product space $\overline{\mathbb{R}}_{+}^{C}$.

As we suppose the bilinear form to be non-singular, the weak upper topology $w(D, C)$ satisfies the $\mathrm{T}_{0}$ separation axiom. The specialization order is given by $y \leq y^{\prime}$ if $\langle x, y\rangle \leq$ $\left\langle x, y^{\prime}\right\rangle$ for all $x \in C$, that is, if $\widehat{y}(x) \leq \widehat{y^{\prime}}(x)$ for all $x \in C$.

Remark 4.1. We may define a weak upper topology $w(D, B)$ for any subset $B$ by taking the coarsest topology rendering continuous the maps $\widehat{x}$ for all $x \in B$. This weak upper topology agrees with the weak upper topology $w(D$, Cone $(B))$, where cone $(B)$ denotes the subcone of $C$ generated by $B$. This follows from the fact that finite linear combinations of lower semicontinuous functionals are also lower semicontinuous.

As the weak upper topology is generated by the subbasic open sets $U_{x}, x \in C$, the finite intersections

$$
U_{F}=\{y \in D \mid\langle x, y\rangle>1 \text { for all } x \in F\}
$$

where $F$ is a finite subset of $C$ form a basis of the weak upper topology. Since these basic open sets are convex, this topology is locally convex in the sense that every point has a neighborhood basis of open convex sets. Every $w(D, C)$-open set is the union of a family of basic open sets $U_{F}$. Translating this statement to the homogeneous functionals according to Lemma 3.3 we obtain:

Lemma 4.2. If $D$ carries the weak upper topology $w(D, C)$, the homogeneous lower semicontinuous functionals $\psi: D \rightarrow \overline{\mathbb{R}}_{+}$are those obtained from the functionals $\widehat{x}, x \in C$, as pointwise sups of finite pointwise infs.

We now come to our general form of Lemma 1.2 .

Theorem 4.3. Let $C, D$ be a pair of cones together with a non-singular bilinear map $C \times D \rightarrow \overline{\mathbb{R}}_{+}$. We equip $D$ with the weak upper topology $w(D, C)$. Then every lower semicontinuous sublinear functional $\psi: D \rightarrow \overline{\mathbb{R}}_{+}$is the pointwise supremum of a family of point evaluations $\widehat{x}$; more explicitly: For every $y \in D$,

$$
\psi(y)=\sup \{\widehat{x}(y) \mid x \in C, \widehat{x} \leq \psi\}=\sup \{\langle x, y\rangle \mid \forall z \in D .\langle x, z\rangle \leq \psi(z)\}
$$

Proof. By the preceding lemma every homogeneous lower semicontinuous functional $\psi$ on $D$ is the pointwise sup of finite pointwise meets $\widehat{x}_{1} \wedge \cdots \wedge \widehat{x}_{n}$ where the $x_{i}$ range over finite families of elements in $C$. If $\psi$ is sublinear, Proposition 2.7 tells us that there is a convex combination $\sum_{i=1}^{n} a_{i} \widehat{x}_{i}, a_{i} \in \mathbb{R}_{+}, \sum_{i} a_{i}=1$, such that $\widehat{x}_{1} \wedge \cdots \wedge \widehat{x}_{n} \leq \sum_{i=1}^{n} a_{i} \widehat{x}_{i} \leq \psi$. Since $\sum_{i=1}^{n} a_{i} \widehat{x}_{i}=\sum_{i=1}^{n} a_{i} x_{i}$, we have found an element $x=\sum_{i=1}^{n} a_{i} x_{i}$ in $C$ with $\widehat{x}_{1} \wedge \cdots \wedge \widehat{x}_{n} \leq$ $\widehat{x} \leq \psi$. Thus $\psi$ is the pointwise sup of functionals $\widehat{x}$.

Concerning the previous proof, note that the functional $\widehat{x}_{1} \wedge \cdots \wedge \widehat{x}_{n}$ is superlinear and lower semicontinuous. Thus the Sandwich Theorem [8, 8.2] allows to find a continuous linear functional $\varphi$ with $\widehat{x}_{1} \wedge \cdots \wedge \widehat{x}_{n} \leq \varphi \leq \psi$. But that is not what we want. We want to show that we can choose $\varphi=\widehat{x}$ for some $x \in C$. For this we use Proposition 2.7 which was proved through a reduction of the problem to the finite dimensional case.

In the following we rephrase our theorem for some special situations. 
4.1. Dual pairs of ordered cones. One specializes the cones $C$ and $D$ above by a dual pair of ordered cones $C$ and $D$ with an order preserving nonsingular bilinear map $(x, y) \mapsto$ $\langle x, y\rangle: C \times D \rightarrow \overline{\mathbb{R}}_{+}$. Note that the given order on $D$ may be stronger than the specialization preorder induced by the weak upper topology $w(D, C)$. Both orders agree if and only if the bilinear form is order non-singular in the sense that for $x \not \leq x^{\prime}$ in $C$ there is a $y \in D$ such that $\langle x, y\rangle \not\left\langle\left\langle x^{\prime}, y\right\rangle\right.$, and similarly for the other argument. Under these hypotheses, Theorem 4.3 specializes to:

Corollary 4.4. For every sublinear functional $\psi: D \rightarrow \overline{\mathbb{R}}_{+}$which is lower semicontinuous with respect to the weak upper topology $w(D, C)$ we have $\psi(y)=\sup \{\widehat{x}(y) \mid x \in C, \widehat{x} \leq \psi\}$ for every $y \in D$.

4.2. Dual pairs of semitopological cones. We now consider a pair $C, D$ of semitopological cones together with a separately continuous non-singular bilinear form $(x, y) \mapsto$ $\langle x, y\rangle: C \times D \rightarrow \overline{\mathbb{R}}_{+}$. The weak upper topology $w(D, C)$ is coarser than the given topology on $D$. Applying Theorem 4.3 under these hypotheses we obtain:

Corollary 4.5. For every sublinear functional $\psi: D \rightarrow \overline{\mathbb{R}}_{+}$which is lower semicontinuous with respect to the weak upper topology $w(D, C)$ we have $\psi(y)=\sup \{\langle x, y\rangle \mid x \in C, \widehat{x} \leq \psi\}$ for every $y \in D$.

Since the given topology on $D$ may be strictly coarser than the weak upper topology $w(D, C)$, the claim of the corollary need not be true for the sublinear functionals $\psi: D \rightarrow \overline{\mathbb{R}}_{+}$ which are lower semicontinuous with respect to the original topology on $D$.

4.3. Dual pairs of d-cones. Recall that a poset $D$ is directed complete (a dcpo, for short), if every directed family $\left(x_{i}\right)_{i}$ of elements in $D$ has a least upper bound, denoted by $\bigvee_{i}^{\uparrow} x_{i}$. A map $f: C \rightarrow D$ between dcpos is called Scott-continuous if it preserves least upper bounds of directed sets, that is, if it is order preserving and if $f\left(\bigvee_{i}^{\uparrow} x_{i}\right)=\bigvee_{i}^{\uparrow} f\left(x_{i}\right)$ for every directed family of elements in $C$. These are precisely the functions that are continuous for the respective Scott topologies, where a subset $A$ of a dcpo is called Scott-closed if $A$ is a lower set and if $\bigvee_{i}^{\uparrow} x_{i} \in A$ for every directed family of $x_{i} \in A$. We refer to [4] for background on dcpos.

A $d$-cone is a cone equipped with directed complete partial order in such a way that scalar multiplication $(r, x) \mapsto r x: \mathbb{R}_{+} \times C \rightarrow C$ and addition $(x, y) \mapsto x+y: C \times C \rightarrow C$ are Scott-continuous. With respect to the Scott topology a d-cone is a semitopological cone. $\overline{\mathbb{R}}_{+}$with its usual order is a d-cone; its Scott topology agrees with the upper topology. Thus the Scott-continuous functionals on a d-cone are the lower semicontinuous ones.

We can specialize our results to dual pairs of d-cones $C, D$ with a Scott-continuous non-singular bilinear form $\langle-,-\rangle: C \times C \rightarrow C$. But here we want to consider also subcones $C_{0} \subseteq C$ which are $d$-dense in $C$, that is, the only d-subcone of $C$ that contains $C_{0}$ is $C$ itself. We have:

Lemma 4.6. The weak upper topology $w\left(D, C_{0}\right)$ agrees with the weak upper topology $w(D, C)$.

Proof. In order to verify our claim, we first notice: If $\left(x_{i}\right)_{i}$ is a directed family in $C$ and $x=$ $\bigvee_{i}^{\uparrow} x_{i}$, then $U_{x}=\bigcup_{i} U_{x_{i}}$. Indeed, $y \in U_{x}$ if and only if $1<\langle x, y\rangle=\left\langle\bigvee_{i}^{\uparrow} x_{i}, y\right\rangle=\bigvee_{i}^{\uparrow}\left\langle x_{i}, y\right\rangle$ (by the Scott-continuity of the bilinear form) if and only if $1<\left\langle x_{i}, y\right\rangle$ for some $i$ if and 
only if $y \in U_{x_{i}}$ for some $i$. This shows that adding suprema of directed sets to the set $C_{0}$ does not refine the weak upper topology. By [10, Corollary 6.7] this suffices to justify our claim.

Under the hypotheses of this subsection, Theorem 4.3 now specializes to the following:

Corollary 4.7. Let $C, D$ be a dual pair of $d$-cones and $C_{0}$ a d-dense subcone of $C$. We endow $D$ with the weak upper topology $w\left(D, C_{0}\right)$ and consider a Scott-continuous sublinear functional $\psi: D \rightarrow \overline{\mathbb{R}}_{+}$. Then $\psi(y)=\sup \left\{\widehat{x}(y) \mid x \in C_{0}, \widehat{x} \leq \psi\right\}$ for every $y \in D$.

\section{Dual Cones And the Schröder-Simpson Theorem}

The standard situation for applying the previous setting is to start with a cone $C$, to form the algebraic dual $D=C^{\prime}$ of all linear functionals $\mu: C \rightarrow \overline{\mathbb{R}}_{+}$which is again a cone under pointwise addition and scalar multiplication and to take the bilinear map $\langle x, \mu\rangle=\mu(x)$ for $x \in C, \mu \in C^{\prime}$ which is non-singular for obvious reasons.

For ordered cones $C$ it is natural to restrict to the order dual of all order preserving linear functionals.

If we start with a semitopological cone $C$, we consider the topological dual $C^{*}$ of all lower semicontinuous linear functionals on $C$ which is a subcone of the algebraic dual $C^{\prime}$. We consider the weak upper topology $w\left(C^{*}, C\right)$ on $C^{*}$ which is also called the weak upper topology in agreement with the terminology in functional analysis. It is the coarsest topology for which the evaluation maps

$$
\widehat{x}=(\mu \mapsto \mu(x)): C^{*} \rightarrow \overline{\mathbb{R}}_{+}
$$

are lower semicontinuous for all $x \in C$. The bilinear map $(x, \mu) \mapsto \mu(x): C \times C^{*} \rightarrow \overline{\mathbb{R}}_{+}$is separately lower semicontinuous. We ask:

Question 5.1. Characterize those semitopological cones $C$ with the property that, for every linear functional $\varphi: C^{*} \rightarrow \overline{\mathbb{R}}_{+}$that is lower semicontinuous for the weak*upper topology, there is a unique element $x \in C$ such that $\varphi(\mu)=\mu(x)$ for every $\mu \in C^{*}$, that is, $\varphi=\widehat{x}$ for a uniquely determined $x \in C$.

Since the evaluation maps are linear, $x \mapsto \widehat{x}$ yields a map from $C$ to the double dual $C^{* *}$ which is linear. The question formulated above is equivalent to the question whether the map $x \mapsto \widehat{x}: C \rightarrow C^{* *}$ is an isomorphism of cones.

A simple example for cones having this property are the 'finite dimensional' cones $\overline{\mathbb{R}}_{+}^{n}$ endowed with the upper topology (which agrees with the product topology with respect to the upper topology on $\overline{\mathbb{R}}_{+}$). This follows from the fact that the dual cone of all lower semicontinuous linear functionals on $\overline{\mathbb{R}}_{+}^{n}$ is isomorphic to $\overline{\mathbb{R}}_{+}^{n}$ and its weak*upper topology agrees with the upper topology. For the proof we just need the following:

Lemma 5.2. 3.2 For every lower semicontinuous linear functional $G: \overline{\mathbb{R}}_{+}^{n} \rightarrow \overline{\mathbb{R}}_{+}$there are $r_{1}, \ldots, r_{n} \in \overline{\mathbb{R}}_{+}$such that $G\left(x_{1}, \ldots, x_{n}\right)=r_{1} x_{1}+\cdots+r_{n} x_{n}$.

Proof. Given $G: \overline{\mathbb{R}}_{+}^{n} \rightarrow \overline{\mathbb{R}}_{+}$, let $r_{i}=G\left(e_{i}\right) \in \overline{\mathbb{R}}_{+}$where $e_{i}$ is the standard basis vector all entries of which are zero, except the i-th entry which is 1 . By linearity, $G\left(x_{1}, \ldots, x_{n}\right)=$ $\sum_{i} r_{i} x_{i}$ for all $\left(x_{1}, \ldots, x_{n}\right) \in \mathbb{R}_{+}^{n}$. By lower semicontinuity this formula extends to all of $\overline{\mathbb{R}}_{+}^{n}$. 
The Schröder-Simpson Theorem yields a whole class of examples for which the answer to Question 5.1 is affirmative. Indeed, for any topological space $X$, the cone $C=\mathcal{L} X$ of lower semicontinuous functions $f: X \rightarrow \overline{\mathbb{R}}_{+}$is a d-cone and its dual is the valuation powerdomain $C^{*}=\mathcal{V} X$.

Of course, Question 5.1 can be split. The injectivity of the map $x \mapsto \widehat{x}$ is equivalent to the question whether the lower semicontinuous linear functionals on $C$ separate the points of $C$. Indeed, for elements $x \neq y$ in $C$, there is a $\mu \in C^{*}$ such that $\mu(x) \neq \mu(y)$ if, and only if, $\widehat{x}(\mu) \neq \widehat{y}(\mu)$, that is, $\widehat{x} \neq \widehat{y}$. This criterion for injectivity is equivalent to the property that, whenever $x \not \leq y$, there is a convex open set $U \subseteq C$ containing $x$ but not $y$. This is a consequence of the Separation Theorem $([8,9.1])$. This property is in particular guaranteed by local convexity: A semitopological cone is locally convex if every point has a neighborhood base of open convex sets. Thus, injectivity is not the problem; surjectivity is the issue.

We are mainly interested in the case of a d-cone $C$ with its Scott topology. We then form the dual cone $D=C^{*}$ of all Scott-continuous linear maps $\mu: C \rightarrow \overline{\mathbb{R}}_{+}$which is a d-cone, too, for the pointwise defined order. The bilinear map $\langle x, \mu\rangle=\mu(x)$ is Scott-continuous. Again, the topology $w\left(C^{*}, C\right)$ on $C^{*}$ is called the weak*upper topology. By Lemma 4.6 above, we can replace $C$ by any d-dense subcone $C_{0}$ since then $w\left(C^{*}, C\right)=w\left(C^{*}, C_{0}\right)$. Theorem 4.3 now specializes to the following general version of Lemma 1.2 in the Introduction:

Corollary 5.3. Let $C$ be a d-cone and $C^{*}$ its dual cone of Scott-continuous linear functionals $\mu: C \rightarrow \overline{\mathbb{R}}_{+}$endowed with its weak ${ }^{*}$-upper topology. Let $C_{0}$ be a d-dense subcone of $C$ in the sense that there is no proper $d$-subcone of $C$ containing $C_{0}$. Then for every lower semicontinuous sublinear functional $\varphi: C^{*} \rightarrow \overline{\mathbb{R}}_{+}$we have $\varphi(\mu)=\sup \left\{\mu(x) \mid x \in C_{0}, \widehat{x} \leq \varphi\right\}$ for every $\mu \in C^{*}$, that is, $\varphi=\sup \left\{\widehat{x} \mid x \in C_{0}, \widehat{x} \leq \varphi\right\}$.

What we are lacking is a general version of Lemma 1.3. So we are left with the question: Characterize the d-cones $C$ for which the conclusion of the previous corollary can be strengthened as follows:

For every lower semicontinuous linear functional $\varphi: C^{*} \rightarrow \overline{\mathbb{R}}_{+}$there is a directed family

$x_{i}$ of elements in $C$ such that $\varphi(\mu)=\bigvee_{i}^{\uparrow} \mu\left(x_{i}\right)$ for all $\mu \in C^{*}$.

If this is the case, then we may form $x=\bigvee_{i}^{\uparrow} x_{i}$ and we obtain $\varphi(\mu)=\bigvee_{i}^{\uparrow} \mu\left(x_{i}\right)=\mu\left(\bigvee_{i}^{\uparrow} x_{i}\right)=$ $\mu(x)$, since all the $\mu \in C^{*}$ are Scott-continuous.

\section{Comments}

The following example may be instructive. Even in the finite dimensional case not every linear functional on a cone with values in $\mathbb{R}_{+}$may be lower semicontinuous for a weak upper topology.

Example 6.1. Take the discrete two elements space $2=\{0,1\}$ and the Sierpinski space $\Sigma$, the two element set $\{0,1\}$ with $\{1\}$ as the only proper nonempty open subset, let $D=\mathcal{L} 2=$ $\mathbb{R}_{+}^{2}$ and $C=\mathcal{L} \Sigma=\left\{\left(x_{1}, x_{2}\right) \in \mathbb{R}_{+}^{2} \mid x_{1} \geq x_{2}\right\}$. The standard inner product is a bilinear map $C \times D \rightarrow \mathbb{R}_{+}$. One might think that this situation gives a counterexample to Theorem 4.3. Indeed, the second projection $\pi_{2}=\left(\left(y_{1}, y_{2}\right) \mapsto y_{2}\right): D \rightarrow \mathbb{R}_{+}$is a linear functional, but there is no $x=\left(x_{1}, x_{2}\right) \in C$ such that $\pi_{2}\left(y_{1}, y_{2}\right)=\left\langle\left(x_{1}, x_{2}\right),\left(y_{1}, y_{2}\right)\right\rangle$. (The only point $x$ satisfying this equation is $x=(0,1)$ which does not belong to $C$.) Also, it is not possible to obtain $\pi_{2}$ as the pointwise sup of functionals of the type $\widehat{x}=(y \mapsto\langle x, y\rangle)$ with $x \in C$. But there is 
not contradiction to Theorem 4.3. The point is, that $\pi_{2}$ is not lower semicontinuous with respect to the weak upper topology $w(D, C)$. Indeed, the specialization order $\leq_{s}$ associated with the topology $w(D, C)$ has to be preserved by functionals that are lower semicontinuous with respect to the weak upper topology $w(D, C)$. In this case the specialization order on $D$ is given by

$$
\left(y_{1}, y_{2}\right) \leq_{s}\left(y_{1}^{\prime}, y_{2}^{\prime}\right) \Longleftrightarrow y_{1} \leq y_{1}^{\prime}, y_{1}+y_{2} \leq y_{1}^{\prime}+y_{2}^{\prime}
$$

The projection $\pi_{2}$ does not preserve $\leq_{s}$, since $(1,1) \leq(2,0)$ but $\pi_{2}(1,1)=1 \not \leq 0=\pi_{2}(2,0)$.

In the following we show a phenomenon that looks surprising:

Example 6.2. Let $C$ and $D$ be subcones of $\mathbb{R}^{n}$ which are closed and have interior points for the usual Euclidean topology. Suppose in addition that, for the standard inner product, $\langle c, d\rangle \geq 0$ for all $c \in C, d \in D$. We claim: $C$ is the dual $D^{*}$ of $D$ in the sense that for every linear functional $\varphi: D \rightarrow \mathbb{R}_{+}$, which is lower semicontinuous for the weak upper topology $w(D, C)$, there is a unique $x \in C$ such that $\varphi=\widehat{x}$ for some $x \in C$.

For the proof consider such a linear functional $\varphi$. It may be extended in a unique way to a linear functional on $\mathbb{R}^{n}$. Thus there is a unique element $x_{0} \in \mathbb{R}^{n}$ such that $\varphi=\widehat{x_{0}}$. By Proposition 4.3. $\left\langle x_{0}, y\right\rangle=\sup _{v \in X}\langle v, y\rangle$ for all $y \in D$, where $X$ is the set of all $v \in C$ such that $\widehat{v} \leq \widehat{x_{0}}$, that is $\langle v, y\rangle \leq\left\langle x_{0}, y\right\rangle$, that is $\left\langle x_{0}-v, y\right\rangle \geq 0$ for all $y \in D$. This amounts to say that $x_{0}-v \in D^{*}$, that is $v \leq_{D^{*}} x$ for the order $\leq_{D^{*}}$ defined by the cone $D^{*}$. This shows that the set $X$ is compact. Clearly, $X$ is convex. If $x_{0} \notin C$, then $x_{0}-X=\left\{x_{0}-x \mid x \in X\right\}$ is compact and convex but does not contain 0 . Take any $y_{0}$ in the interior of $D$. Then $\widehat{y_{0}}$ is a strictly positive functional on $D^{*}$, that is, $\left\langle x, y_{0}\right\rangle>0$ for all $x \neq 0$ in $D^{*}$. Hence, $\left\langle x-v, y_{0}\right\rangle>0$ for all $v \in X$. Since $X$ is compact, it follows that $\sup _{v \in X}\left\langle v, y_{0}\right\rangle\left\langle\left\langle x, y_{0}\right\rangle\right.$, a contradiction.

Similar results as above hold for d-subcones of $\overline{\mathbb{R}}_{+}^{n}$. But note that $C$ is not the dual of $D$, in general, if we use the usual Euclidean topology on $D$.

\section{REFERENCES}

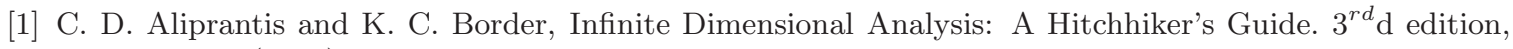
Springer Verlag (2006).

[2] I. Battenfeld, M. Schröder and A. Simpson, A convenient category of domains. In: Computation, Meaning and Logic, Articles dedicated to Gordon Plotkin L. Cardelli, M. Fiore and G. Winskel (eds), Electronic Notes in Computer Science 172 (2007), pages 69-99.

[3] K. C. Border, Separating Hyperplane Theorems. Notes, California Institute of Technology. Www.hss.caltech.edu/ kcb/Notes/SeparatingHyperplane.pdf

[4] G. Gierz, K. H. Hofmann, K. Keimel, J. D. Lawson, M. Mislove, and D. S. Scott, Continuous Lattices and Domains. Encyclopedia of Mathematics and its Applications, vol. 93, Cambridge University Press, 2003, xxxvi+591 pages.

[5] J. Goubault-Larrecq, A short proof of the Schröder-Simpson theorem. Mathematical Structures in Computer Science 25 (2015), pages 1-5.

[6] J. Goubault-Larrecq, Isomorphism Theorems between Models of Mixed Choice, Draft.

[7] C. Jones, Probabilistic non-determinism. PhD Thesis, Department of Computer Science, The University of Edinburgh (1990), 201 pages (also published as Technical Report No. CST-63-90).

[8] K. Keimel, Topological Cones: Functional analysis in a $\mathrm{T}_{0}$-setting. Semigroup Forum 77 (2008), pages 108-142.

[9] K. Keimel, Locally convex cones and the Schröder-Simpson Theorem. Quaestiones Mathematicae 35 (2012), pages 353-390. 
[10] K. Keimel and J. D. Lawson, Extending algebraic operations to D-completions. Theoretical Computer Science 430 (2012), pages 73-87.

[11] O. Kirch, Bereiche und Bewertungen. MSc Thesis, TU Darmstadt, 1993. http://www.mathematik.tu-darmstadt.de/fbereiche/logik/research/Domains/Domains.html

[12] G. D. Plotkin, A domain-theoretic Banach-Alaoglu theorem. Mathematical Structures in Computer Science 16 (2006), pages 299-312.

[13] W. Rudin, Functional Analysis. McGraw-Hill Book Company, 1973.

[14] H. H. Schaefer, Topological Vector Spaces. Springer Verlag, 3rd printing (1970).

[15] M. Schröder and A. Simpson, Probabilistic observations and valuations. Electronic Notes in Theoretical Computer Science 155 (2006), 605-615.

[16] M. Schröder and A. Simpson, Probabilistic Observations and Valuations. Talk at New Interactions Between Analysis, Topology and Computation, Birmingham (2009). http://homepages.inf.ed.ac.uk/als/Talks/birmingham09.pdf 